MATHEMATICS OF COMPUTATION

Volume 81, Number 278, April 2012, Pages 643-659

S $0025-5718(2011) 02540-8$

Article electronically published on August 30, 2011

\title{
HODGE DECOMPOSITION FOR DIVERGENCE-FREE VECTOR FIELDS AND TWO-DIMENSIONAL MAXWELL'S EQUATIONS
}

\author{
S. C. BRENNER, J. CUI, Z. NAN, AND L.-Y. SUNG
}

\begin{abstract}
We propose a new numerical approach for two-dimensional Maxwell's equations that is based on the Hodge decomposition for divergencefree vector fields. In this approach an approximate solution for Maxwell's equations can be obtained by solving standard second order scalar elliptic boundary value problems. This new approach is illustrated by a $P_{1}$ finite element method.
\end{abstract}

\section{INTRODUCTION}

Let $\Omega$ be a (connected) bounded polygonal domain in $\mathbb{R}^{2}, \boldsymbol{f} \in\left[L_{2}(\Omega)\right]^{2}$, and $\mu, \epsilon$ be positive functions in $C^{1}(\bar{\Omega})$. Consider the problem of finding $\boldsymbol{u} \in H_{0}(\operatorname{curl} ; \Omega) \cap$ $H\left(\operatorname{div}^{0} ; \Omega ; \epsilon\right)$ such that

$$
\left(\mu^{-1} \nabla \times \boldsymbol{u}, \nabla \times \boldsymbol{v}\right)+\alpha(\epsilon \boldsymbol{u}, \boldsymbol{v})=(\boldsymbol{f}, \boldsymbol{v}) \quad \forall \boldsymbol{v} \in H_{0}(\operatorname{curl} ; \Omega) \cap H\left(\operatorname{div}^{0} ; \Omega ; \epsilon\right),
$$

where $(\cdot, \cdot)$ denotes the $L_{2}$ inner product, and the spaces $H_{0}(\operatorname{curl} ; \Omega)$ and $H\left(\operatorname{div}^{0} ; \Omega ; \epsilon\right)$ are defined as follows:

$$
\begin{aligned}
H(\operatorname{curl} ; \Omega) & =\left\{\boldsymbol{v}=\left[\begin{array}{l}
v_{1} \\
v_{2}
\end{array}\right] \in\left[L_{2}(\Omega)\right]^{2}: \nabla \times \boldsymbol{v}=\frac{\partial v_{2}}{\partial x_{1}}-\frac{\partial v_{1}}{\partial x_{2}} \in L_{2}(\Omega)\right\}, \\
H_{0}(\operatorname{curl} ; \Omega) & =\{\boldsymbol{v} \in H(\operatorname{curl} ; \Omega): \boldsymbol{n} \times \boldsymbol{v}=0 \text { on } \partial \Omega\},
\end{aligned}
$$

where $\boldsymbol{n}$ is the outward pointing unit normal along $\partial \Omega$,

$$
\begin{aligned}
H(\operatorname{div} ; \Omega) & =\left\{\boldsymbol{v}=\left[\begin{array}{l}
v_{1} \\
v_{2}
\end{array}\right] \in\left[L_{2}(\Omega)\right]^{2}: \nabla \cdot \boldsymbol{v}=\frac{\partial v_{1}}{\partial x_{1}}+\frac{\partial v_{2}}{\partial x_{2}} \in L_{2}(\Omega)\right\}, \\
H\left(\operatorname{div}^{0} ; \Omega\right) & =\{\boldsymbol{v} \in H(\operatorname{div} ; \Omega): \nabla \cdot \boldsymbol{v}=0\},
\end{aligned}
$$

and

$$
H\left(\operatorname{div}^{0} ; \Omega ; \epsilon\right)=\left\{\boldsymbol{v} \in\left[L_{2}(\Omega)\right]^{2}: \epsilon \boldsymbol{v} \in H\left(\operatorname{div}^{0} ; \Omega\right)\right\} .
$$

Received by the editor January 11, 2010 and, in revised form, March 1, 2011.

2010 Mathematics Subject Classification. Primary 65N30, 65N15, 35Q60.

Key words and phrases. Maxwell's equations, Hodge decomposition, finite element.

This work was supported in part by the National Science Foundation under Grant Nos. DMS07-13835 and DMS-10-16332, and by the Institute for Mathematics and its Applications with funds provided by the National Science Foundation. 
Remark 1.1. $H\left(\operatorname{div}^{0} ; \Omega ; 1\right)$ is identical with $H\left(\operatorname{div}^{0} ; \Omega\right) . H\left(\operatorname{div}^{0} ; \Omega ; \epsilon\right)$ is the orthogonal complement of $\nabla H_{0}^{1}(\Omega)$ in $\left[L_{2}(\Omega ; \epsilon)\right]^{2}$, which is the space of square integrable vector fields equipped with the weighted inner product

$$
(\boldsymbol{v}, \boldsymbol{w})_{L_{2}(\Omega ; \epsilon)}=\int_{\Omega} \epsilon(\boldsymbol{v} \cdot \boldsymbol{w}) d x .
$$

The problem (1.1) is related to the time-harmonic Maxwell's equations for $\alpha \leq 0$ and the time-domain Maxwell's equations for $\alpha>0$ (cf. [16, 19, 10, 6, 7, 8]), where $\mu$ and $\epsilon$ are, respectively, the permeability and permittivity. We assume that (1.1) is uniquely solvable, i.e., $-\alpha$ is not a Maxwell eigenvalue. In particular, we assume $\alpha \neq 0$ when $\Omega$ is not simply connected.

In this paper we develop a new numerical approach to (1.1) using the Hodge decomposition of $\boldsymbol{u}$. In this approach an approximation of $\boldsymbol{u}$ can be obtained by solving standard second order scalar elliptic boundary value problems.

More precisely, we use the Hodge decomposition for $H\left(\operatorname{div}^{0} ; \Omega ; \epsilon\right)$ to write

$$
\boldsymbol{u}=\epsilon^{-1} \nabla \times \phi+\sum_{j=1}^{m} c_{j} \nabla \varphi_{j},
$$

where $\phi \in H^{1}(\Omega)$ satisfies $(\phi, 1)=0$,

$$
\nabla \times \phi=\left[\begin{array}{r}
\frac{\partial \phi}{\partial x_{2}} \\
-\frac{\partial \phi}{\partial x_{1}}
\end{array}\right],
$$

the nonnegative integer $m$ is the Betti number for $\Omega(m=0$ if $\Omega$ is simply connected), and the functions $\varphi_{1}, \ldots, \varphi_{m}$ are defined as follows.

Let the outer boundary of $\Omega$ be denoted by $\Gamma_{0}$ and the $m$ components of the inner boundary be denoted by $\Gamma_{1}, \ldots, \Gamma_{m}$. Then the functions $\varphi_{j}$ are determined by

$$
\begin{array}{rlrl}
\left(\epsilon \nabla \varphi_{j}, \nabla v\right) & =0 & & \forall v \in H_{0}^{1}(\Omega), \\
\left.\varphi_{j}\right|_{\Gamma_{0}}=0, & \text { for } 1 \leq k \leq m . \\
\left.\varphi_{j}\right|_{\Gamma_{k}}=\delta_{j k}= \begin{cases}1 & j=k, \\
0 & j \neq k,\end{cases}
\end{array}
$$

We will show that the function $\phi$ in (1.2) is determined by

$$
\left(\nabla \times \phi, \epsilon^{-1} \nabla \times \psi\right)=(\mu \xi, \psi) \quad \forall \psi \in H^{1}(\Omega)
$$

and the constraint

$$
(\phi, 1)=0
$$

where the function $\xi=\mu^{-1} \nabla \times \boldsymbol{u} \in H^{1}(\Omega)$ is determined by

$$
\left(\nabla \times \xi, \epsilon^{-1} \nabla \times \psi\right)+\alpha(\mu \xi, \psi)=\left(\boldsymbol{f}, \epsilon^{-1} \nabla \times \psi\right) \quad \forall \psi \in H^{1}(\Omega)
$$

when $\alpha \neq 0$, and by (1.6) together with the constraint

$$
(\mu \xi, 1)=0
$$

when $\Omega$ is simply connected and $\alpha=0$. 
Note that $\alpha \neq 0$ when $m \geq 1$ since 0 is a Maxwell eigenvalue for domains that are not simply connected. In this case we will show that the coefficients $c_{j}$ in (1.2) are determined by the symmetric positive-definite system

$$
\sum_{j=1}^{m}\left(\epsilon \nabla \varphi_{j}, \nabla \varphi_{k}\right) c_{j}=\frac{1}{\alpha}\left(\boldsymbol{f}, \nabla \varphi_{k}\right) \quad \text { for } \quad 1 \leq k \leq m .
$$

We can therefore solve (1.1) by the following procedure.

(1) Compute a numerical approximation $\tilde{\xi}$ of $\xi$ by solving (1.6) when $\alpha \neq 0$, and by solving (1.6) with the constraint (1.7) when $\Omega$ is simply connected and $\alpha=0$.

(2) Compute a numerical approximation $\tilde{\phi}$ of $\phi$ by solving (1.4) under the constraint (1.5), where $\xi$ is replaced by $\tilde{\xi}$.

(3) Compute numerical approximations $\tilde{\varphi}_{1}, \ldots, \tilde{\varphi}_{m}$ of $\varphi_{1}, \ldots, \varphi_{m}$ by solving the boundary value problems in (1.3).

(4) Compute numerical approximations $\tilde{c}_{1}, \ldots, \tilde{c}_{m}$ of $c_{1}, \ldots, c_{m}$ by solving (1.8), where $\varphi_{1}, \ldots, \varphi_{m}$ are replaced by $\tilde{\varphi}_{1}, \ldots, \tilde{\varphi}_{m}$.

(5) The numerical approximation $\tilde{\boldsymbol{u}}$ for $\boldsymbol{u}$ is given by

$$
\tilde{\boldsymbol{u}}=\epsilon^{-1} \nabla \times \tilde{\phi}+\sum_{j=1}^{m} \tilde{c}_{j} \nabla \tilde{\varphi}_{j} .
$$

Remark 1.2. The function $\tilde{\xi}$ computed in Step (1) provides an approximation for $\mu^{-1} \nabla \times \boldsymbol{u}$.

Remark 1.3. Since the functions $\varphi_{j}$ depend only on the domain $\Omega$, Step (3) can be carried out once $\Omega$ is given. Hence the solution of (1.1) is essentially reduced to the solution of the two elliptic boundary value problems in Steps (1)-(2).

Remark 1.4. The equations (1.4) and (1.6) can be rewritten as

$$
\begin{aligned}
\left(\nabla \phi, \epsilon^{-1} \nabla \psi\right) & =(\mu \xi, \psi) & & \forall \psi \in H^{1}(\Omega), \\
\left(\nabla \xi, \epsilon^{-1} \nabla \psi\right)+\alpha(\mu \xi, \psi) & =\left(\boldsymbol{f}, \epsilon^{-1} \nabla \times \psi\right) & & \forall \psi \in H^{1}(\Omega) .
\end{aligned}
$$

Hence the boundary value problems for $\phi$ and $\xi$ are Neumann problems for the Laplace operator.

Remark 1.5. The Hodge decomposition has also been applied to other electromagnetic problems [2, 3, 5].

Since the boundary value problems in Steps (1)-(3) are standard second order scalar elliptic boundary value problems, they can be solved by many methods. For simplicity, we will demonstrate this new numerical approach by a $P_{1}$ finite element method.

The rest of the paper is organized as follows. We first provide detailed justifications of the new approach in Sections 2 and 3. Then in Section 4 we discuss a numerical method for (1.1) based on $P_{1}$ finite elements. We end with some concluding remarks in Section [5.

For convenience in later sections we state here two useful facts concerning $H(\operatorname{curl} ; \Omega)$ and $H(\operatorname{div} ; \Omega)$. The first is that (cf. [14, Theorems 2.11 and 2.12]) $(1.9)$

$\boldsymbol{v} \in H(\operatorname{curl} ; \Omega)$ belongs to $H_{0}(\operatorname{curl} ; \Omega) \quad$ iff $\quad(\nabla \times \boldsymbol{v}, \psi)=(\boldsymbol{v}, \nabla \times \psi) \quad \forall \psi \in H^{1}(\Omega)$. 
The second is that (cf. [14, Theorem 2.5]) the normal trace $\boldsymbol{n} \cdot \boldsymbol{v} \in H^{-1 / 2}(\partial \Omega)$ is well defined for $\boldsymbol{v} \in H(\operatorname{div} ; \Omega)$. Moreover, we have

$$
(\boldsymbol{v}, \nabla \zeta)+(\nabla \cdot \boldsymbol{v}, \zeta)=\int_{\partial \Omega} \zeta \boldsymbol{n} \cdot \boldsymbol{v} d s \quad \forall \boldsymbol{v} \in H(\operatorname{div} ; \Omega), \zeta \in H^{1}(\Omega) .
$$

\section{Hodge DeCOMPosition}

Discussions of Hodge decompositions for three-dimensional vector fields can be found, for example, in [4, 19]. To make the present article more self-contained we provide here a derivation of the Hodge decomposition for two-dimensional vector fields and justify equations (1.4) and (1.8).

Recall that $\Gamma_{0}$ is the outer boundary of $\Omega$ and $\Gamma_{1}, \ldots, \Gamma_{m}$ are the components of the inner boundary of $\Omega$. The following result (cf. [14, Theorem 3.1]) is crucial for the derivation of the Hodge decomposition.

Lemma 2.1. Let $\boldsymbol{w} \in\left[L_{2}(\Omega)\right]^{2}$. There exists $\phi \in H^{1}(\Omega)$ such that

$$
\boldsymbol{w}=\nabla \times \phi
$$

if and only if $\boldsymbol{w} \in H\left(\operatorname{div}^{0} ; \Omega\right)$ and

$$
\int_{\Gamma_{j}} \boldsymbol{w} \cdot \boldsymbol{n} d s=0 \quad \text { for } \quad 0 \leq j \leq m .
$$

Let $\mathcal{H}(\Omega ; \epsilon)$ be the space of $\epsilon$-harmonic functions spanned by the functions $\varphi_{1}, \ldots, \varphi_{m}$ defined in (1.3). Note that, by the elliptic regularity theory on nonsmooth domains (cf. [15, 13, 20]), there exists $\tau \in(1 / 2,1]$ such that $\varphi_{j} \in H^{1+\tau}(\Omega)$ for $1 \leq j \leq m$.

Lemma 2.2. Let $\varphi \in \mathcal{H}(\Omega ; \epsilon)$. Then

$$
\int_{\Gamma_{j}} \epsilon \frac{\partial \varphi}{\partial n} d s=0 \quad \text { for } \quad 1 \leq j \leq m
$$

if and only if $\varphi=0$.

Proof. Let $\varphi=\sum_{j=1}^{m} b_{j} \varphi_{j}$ satisfy (2.1). It follows from (1.3) that

$$
(\epsilon \nabla \varphi, \nabla \varphi)=\sum_{j=1}^{m} \int_{\Gamma_{j}} \epsilon \frac{\partial \varphi}{\partial n} \varphi d s=\sum_{j=1}^{m} b_{j} \int_{\Gamma_{j}} \epsilon \frac{\partial \varphi}{\partial n} d s=0
$$

which implies $\varphi=0$ since $\varphi$ vanishes on the outer boundary $\Gamma_{0}$.

Lemma 2.3. Given any $\boldsymbol{v} \in H\left(\operatorname{div}^{0} ; \Omega ; \epsilon\right)$, there exist a unique $\phi \in H^{1}(\Omega)$ and $m$ unique real numbers $c_{1}, \ldots, c_{m}$ such that $(\phi, 1)=0$ and

$$
\boldsymbol{v}=\epsilon^{-1} \nabla \times \phi+\sum_{j=1}^{m} c_{j} \nabla \varphi_{j}
$$

Proof. It follows from Lemma 2.2 that the map

$$
\varphi \longrightarrow\left[\begin{array}{c}
\int_{\Gamma_{1}} \epsilon(\partial \varphi / \partial n) d s \\
\vdots \\
\int_{\Gamma_{m}} \epsilon(\partial \varphi / \partial n) d s
\end{array}\right]
$$


is an isomorphism from $\mathcal{H}(\Omega ; \epsilon)$ into $\mathbb{R}^{m}$. Therefore, there exist unique constants $c_{1}, \ldots, c_{m}$ such that

$$
\int_{\Gamma_{k}} \epsilon\left(\boldsymbol{v}-\sum_{j=1}^{m} c_{j} \nabla \varphi_{j}\right) \cdot \boldsymbol{n} d s=0 \quad \text { for } 1 \leq k \leq m .
$$

Since $\epsilon \boldsymbol{v} \in H\left(\operatorname{div}^{0} ; \Omega\right)$ by the definition of $H\left(\operatorname{div}^{0} ; \Omega ; \epsilon\right)$ and $\epsilon \nabla \varphi_{j} \in H\left(\operatorname{div}^{0} ; \Omega\right)$ by construction (cf. (1.3a)), we can apply (1.10) with $\zeta=1$ to obtain

$$
\int_{\partial \Omega} \epsilon\left(\boldsymbol{v}-\sum_{j=1}^{m} c_{j} \nabla \varphi_{j}\right) \cdot \boldsymbol{n} d s=0
$$

which together with (2.3) implies

$$
\int_{\Gamma_{0}} \epsilon\left(\boldsymbol{v}-\sum_{j=1}^{m} c_{j} \nabla \varphi_{j}\right) \cdot \boldsymbol{n} d s=0 .
$$

It then follows from Lemma 2.1 and (2.3) $-(2.4)$ that

$$
\epsilon\left(\boldsymbol{v}-\sum_{j=1}^{m} c_{j} \nabla \varphi_{j}\right)=\nabla \times \phi
$$

for some $\phi \in H^{1}(\Omega)$, which of course can be chosen to satisfy the constraint $(\phi, 1)=0$.

The uniqueness of the decomposition (2.2) follows from the fact (cf. Lemma 2.1) that (2.3) must hold for any such decomposition, which implies that the coefficients $c_{1}, \ldots, c_{m}$ are unique. The uniqueness of $\phi$ then follows immediately.

We need the following lemma for the derivation of (1.4) and (1.8).

Lemma 2.4. Let $\zeta \in H^{1}(\Omega)$ such that the trace of $\zeta$ on $\Gamma_{j}$ is a constant $\gamma_{j}$ for $0 \leq j \leq m$. Then we have

$$
(\nabla \times \psi, \nabla \zeta)=0 \quad \forall \psi \in H^{1}(\Omega) .
$$

Proof. From Lemma 2.1, we have

$$
\int_{\Gamma_{j}} \boldsymbol{n} \cdot(\nabla \times \psi) d s=0 \quad \text { for } \quad 0 \leq j \leq m,
$$

which together with (1.10) (where $\boldsymbol{v}$ is taken to be $\nabla \times \psi$ ) implies

$$
(\nabla \times \psi, \nabla \zeta)=\sum_{j=0}^{m} \int_{\Gamma_{j}} \zeta \boldsymbol{n} \cdot(\nabla \times \psi) d s=\sum_{j=0}^{m} \gamma_{j} \int_{\Gamma_{j}} \boldsymbol{n} \cdot(\nabla \times \psi) d s=0 .
$$

Note that (1.9) (with $\boldsymbol{v}=\nabla \zeta$ ) and (2.5) imply that $\nabla \zeta \in H_{0}(\operatorname{curl} ; \Omega)$ for any $\zeta$ satisfying the assumptions of Lemma 2.4. The following corollary is therefore immediate.

Corollary 2.5. We have $\nabla H_{0}^{1}(\Omega) \subset H_{0}(\operatorname{curl} ; \Omega)$ and $\nabla \mathcal{H}(\Omega ; \epsilon) \subset H_{0}(\operatorname{curl} ; \Omega) \cap$ $H\left(\operatorname{div}^{0} ; \Omega ; \epsilon\right)$.

Remark 2.6. Because of Corollary 2.5, the decomposition (2.2) can be viewed as a decomposition in $H_{0}(\operatorname{curl} ; \Omega) \cap H\left(\operatorname{div}^{0} ; \Omega ; \epsilon\right)$ if $\boldsymbol{v} \in H_{0}(\operatorname{curl} ; \Omega) \cap H\left(\operatorname{div}^{0} ; \Omega ; \epsilon\right)$. In this case $\phi$ has higher regularity and $\partial \phi / \partial n=0$ on $\partial \Omega$.

Remark 2.7. There are other decompositions of $H_{0}(\operatorname{curl} ; \Omega) \cap H\left(\operatorname{div}^{0} ; \Omega\right)$ that can be exploited for the purpose of preconditioning $H(\operatorname{curl} ; \Omega)$ conforming methods [22, 17. 
We can now use (1.2), (1.9) and Lemma 2.4 to justify (1.4) as follows. Let $\psi \in H^{1}(\Omega)$ be arbitrary. We have

$$
\begin{aligned}
\left(\nabla \times \phi, \epsilon^{-1} \nabla \times \psi\right) & =\left(\nabla \times \phi+\epsilon \sum_{j=1}^{m} c_{j} \nabla \varphi_{j}, \epsilon^{-1} \nabla \times \psi\right) \\
& =\left(\epsilon \boldsymbol{u}, \epsilon^{-1} \nabla \times \psi\right)=(\boldsymbol{u}, \nabla \times \psi)=(\nabla \times \boldsymbol{u}, \psi)=(\mu \xi, \psi) .
\end{aligned}
$$

To justify (1.8) when $m \geq 1$, we take $\boldsymbol{v}=\nabla \varphi_{k}$ in (1.1) (cf. Corollary 2.5) and replace $\boldsymbol{u}$ by the Hodge decomposition (1.2). We arrive at the equation

$$
\alpha\left(\nabla \times \phi+\epsilon \sum_{j=1}^{m} c_{j} \nabla \varphi_{j}, \nabla \varphi_{k}\right)=\left(\boldsymbol{f}, \nabla \varphi_{k}\right) \quad \text { for } \quad 1 \leq k \leq m,
$$

which together with Lemma 2.4 implies (1.8).

Finally, we observe that the bilinear form $(\varphi, \varrho) \rightarrow(\epsilon \nabla \varphi, \nabla \varrho)$ is symmetric positive-definite on $\mathcal{H}(\Omega ; \epsilon)$, because $(\epsilon \nabla \varphi, \nabla \varphi)=0$ implies $\varphi=0$ since $\varphi$ vanishes on the outer boundary $\Gamma_{0}$ of $\Omega$. Hence the system (1.8) is symmetric positivedefinite.

\section{Equation For $\xi=\mu^{-1} \nabla \times u$}

In this section we derive the equation (1.6). We begin with the strong form of (1.1).

Lemma 3.1. The solution $\boldsymbol{u}$ of (1.1) satisfies

$$
\nabla \times\left(\mu^{-1} \nabla \times \boldsymbol{u}\right)+\alpha(\epsilon \boldsymbol{u})=\epsilon Q\left(\epsilon^{-1} \boldsymbol{f}\right)
$$

in the sense of distributions, where $Q:\left[L_{2}(\Omega ; \epsilon)\right]^{2} \longrightarrow H\left(\operatorname{div}^{0} ; \Omega ; \epsilon\right)$ is the orthogonal projection.

Proof. Let $\zeta \in\left[C_{c}^{\infty}(\Omega)\right]^{2}$ be a $C^{\infty}$ vector field with compact support in $\Omega$. We have $\boldsymbol{\zeta} \in H_{0}(\operatorname{curl} ; \Omega), Q \boldsymbol{\zeta} \in H\left(\operatorname{div}^{0} ; \Omega ; \epsilon\right)$ and $\boldsymbol{\zeta}-Q \boldsymbol{\zeta}=\nabla H_{0}^{1}(\Omega)$ (cf. Remark 1.1).

Since $\nabla H_{0}^{1}(\Omega) \subset H_{0}(\operatorname{curl} ; \Omega)$ (cf. Corollary 2.5), we have $\boldsymbol{\zeta}-Q \boldsymbol{\zeta} \in H_{0}(\operatorname{curl} ; \Omega)$ and hence $Q \boldsymbol{\zeta}=\boldsymbol{\zeta}-(\boldsymbol{\zeta}-Q \boldsymbol{\zeta}) \in H_{0}(\operatorname{curl} ; \Omega)$. It follows that

$$
Q \zeta \in H_{0}(\operatorname{curl} ; \Omega) \cap H\left(\operatorname{div}^{0} ; \Omega ; \epsilon\right) .
$$

Furthermore, from $\nabla \times\left(\nabla H_{0}^{1}(\Omega)\right)=\{0\}$ and $\boldsymbol{u} \in H\left(\operatorname{div}^{0} ; \Omega ; \epsilon\right)$, we have

$$
\nabla \times(\boldsymbol{\zeta}-Q \boldsymbol{\zeta})=0=(\boldsymbol{u}, \boldsymbol{\zeta}-Q \boldsymbol{\zeta})_{L_{2}(\Omega ; \epsilon)}=(\epsilon \boldsymbol{u}, \boldsymbol{\zeta}-Q \boldsymbol{\zeta}) .
$$

Using (1.1), (3.2) and (3.3), we can complete the proof of the lemma as follows:

$$
\begin{aligned}
\left(\mu^{-1}\right. & \nabla \times \boldsymbol{u}, \nabla \times \boldsymbol{\zeta})+\alpha(\epsilon \boldsymbol{u}, \boldsymbol{\zeta})=\left(\mu^{-1} \nabla \times \boldsymbol{u}, \nabla \times(Q \boldsymbol{\zeta}+(\boldsymbol{\zeta}-Q \boldsymbol{\zeta}))\right. \\
& +\alpha(\epsilon \boldsymbol{u}, Q \boldsymbol{\zeta}+(\boldsymbol{\zeta}-Q \boldsymbol{\zeta})) \\
& =\left(\mu^{-1} \nabla \times \boldsymbol{u}, \nabla \times Q \boldsymbol{\zeta}\right)+\alpha(\epsilon \boldsymbol{u}, Q \boldsymbol{\zeta}) \\
& =(\boldsymbol{f}, Q \boldsymbol{\zeta})=\left(\epsilon^{-1} \boldsymbol{f}, Q \boldsymbol{\zeta}\right)_{L_{2}(\Omega ; \epsilon)}=\left(Q\left(\epsilon^{-1} \boldsymbol{f}\right), \boldsymbol{\zeta}\right)_{L_{2}(\Omega ; \epsilon)}=\left(\epsilon Q\left(\epsilon^{-1} \boldsymbol{f}\right), \boldsymbol{\zeta}\right) .
\end{aligned}
$$

As a corollary, we have $\xi=\mu^{-1} \nabla \times \boldsymbol{u} \in H^{1}(\Omega)$ and

$$
\nabla \times \xi+\alpha \epsilon \boldsymbol{u}=\epsilon\left(Q \epsilon^{-1} \boldsymbol{f}\right) .
$$


Let $\psi \in H^{1}(\Omega)$ be arbitrary. We have (cf. Lemma 2.1) $\epsilon^{-1} \nabla \times \psi \in H\left(\operatorname{div}^{0} ; \Omega ; \epsilon\right)$, which together with (1.9) and (3.4) implies that

$$
\begin{aligned}
\left(\boldsymbol{f}, \epsilon^{-1} \nabla \times \psi\right) & =\left(\epsilon^{-1} \boldsymbol{f}, \epsilon^{-1} \nabla \times \psi\right)_{L_{2}(\Omega ; \epsilon)} \\
& =\left(Q\left(\epsilon^{-1} \boldsymbol{f}\right), \epsilon^{-1} \nabla \times \psi\right)_{L_{2}(\Omega ; \epsilon)} \\
& =\left(\epsilon Q\left(\epsilon^{-1} \boldsymbol{f}\right), \epsilon^{-1} \nabla \times \psi\right) \\
& =\left(\nabla \times \xi+\alpha(\epsilon \boldsymbol{u}), \epsilon^{-1} \nabla \times \psi\right) \\
& =\left(\nabla \times \xi, \epsilon^{-1} \nabla \times \psi\right)+\alpha(\nabla \times \boldsymbol{u}, \psi)=\left(\nabla \times \xi, \epsilon^{-1} \nabla \times \psi\right)+\alpha(\mu \xi, \psi),
\end{aligned}
$$

i.e., equation (1.6) is valid. The constraint (1.7) follows immediately from (1.9).

Next, we turn to a relation between the solvability of (1.1) and the solvability of (1.6) that will guarantee the well-posedness of (1.6) under the condition that $-\alpha$ $(\neq 0)$ is not a Maxwell eigenvalue. Note that a discussion on the relation between the Maxwell eigenvalues and Laplace eigenvalues can also be found in [12.

Lemma 3.2. For $\alpha \neq 0$, the problem (1.1) is uniquely solvable if and only if the problem (1.6) is uniquely solvable.

Proof. Let $\alpha$ be nonzero. Since $H^{1}(\Omega)$ is compactly embedded in $L_{2}(\Omega)$ by the Rellich-Kondrachov theorem [1] and $H_{0}(\operatorname{curl} ; \Omega) \cap H\left(\operatorname{div}^{0} ; \Omega ; \epsilon\right)$ is compactly embedded in $\left[L_{2}(\Omega)\right]^{2}$ by a result of Weber [25], we can apply the Fredholm alternative to consider only the homogeneous equation corresponding to (1.1)

$$
\left(\mu^{-1} \nabla \times \boldsymbol{w}, \nabla \times \boldsymbol{v}\right)+\alpha(\epsilon \boldsymbol{w}, \boldsymbol{v})=0 \quad \forall \boldsymbol{v} \in H_{0}(\operatorname{curl} ; \Omega) \cap H\left(\operatorname{div}^{0} ; \Omega\right),
$$

and the homogeneous equation corresponding to (1.6)

$$
\left(\nabla \times \eta, \epsilon^{-1} \nabla \times \psi\right)+\alpha(\mu \eta, \psi)=0 \quad \forall \psi \in H^{1}(\Omega) .
$$

We will show that (3.5) has a nontrivial solution $\boldsymbol{w} \in H_{0}(\operatorname{curl} ; \Omega) \cap H\left(\operatorname{div}^{0} ; \Omega ; \epsilon\right)$ if and only if (3.6) has a nontrivial solution $\eta \in H^{1}(\Omega)$.

Suppose there exists a nontrivial $\boldsymbol{w} \in H_{0}(\operatorname{curl} ; \Omega) \cap H\left(\operatorname{div}^{0} ; \Omega ; \epsilon\right)$ that satisfies (3.5) and $\eta=\mu^{-1} \nabla \times \boldsymbol{w}$. Then $\eta \in H^{1}(\Omega)$ and (3.6) holds as a special case of (1.6) where $\boldsymbol{f}=\mathbf{0}$. Moreover, it follows from the Poincaré-Friedrichs inequality (cf. [19, Corollary 4.8])

$$
\|\boldsymbol{w}\|_{L_{2}(\Omega)} \leq C_{\Omega, \epsilon}\|\nabla \times \boldsymbol{w}\|_{L_{2}(\Omega)}=C_{\Omega, \epsilon}\|\mu \eta\|_{L_{2}(\Omega)}
$$

that $\eta \neq 0$.

Suppose there exists a nontrivial $\eta \in H^{1}(\Omega)$ that satisfies (3.6). Since $\alpha \neq 0$, we deduce from (3.6) that $(\mu \eta, 1)=0$. Hence we can write (cf. [14, Corollary 2.4])

$$
\mu \eta=\nabla \times \boldsymbol{w}
$$

for some $\boldsymbol{w} \in H_{0}(\operatorname{curl} ; \Omega) \cap H\left(\operatorname{div}^{0} ; \Omega ; \epsilon\right)$. Indeed, we can take $\boldsymbol{w}=\epsilon^{-1} \nabla \times \rho$, where $\rho \in H^{1}(\Omega)$ is defined by the Neumann problem

$$
\begin{aligned}
\left(\epsilon^{-1} \nabla \times \rho, \nabla \times \psi\right) & =(\mu \eta, \psi) \quad \forall \psi \in H^{1}(\Omega), \\
(\rho, 1) & =0 .
\end{aligned}
$$

Then (3.7) follows from (3.8a) and $\boldsymbol{w} \in H\left(\operatorname{div}^{0} ; \Omega ; \epsilon\right)$ by Lemma 2.1. Since (3.8a) can be written as

$$
(\boldsymbol{w}, \nabla \times \psi)=(\nabla \times \boldsymbol{w}, \psi) \quad \forall \psi \in H^{1}(\Omega),
$$

we also have $\boldsymbol{w} \in H_{0}(\operatorname{curl} ; \Omega)$ by (1.9). It follows that $\boldsymbol{w} \in H_{0}(\operatorname{curl} ; \Omega) \cap H\left(\operatorname{div}^{0} ; \Omega ; \epsilon\right)$. 
Clearly, $\boldsymbol{w}$ is nontrivial. To see that it satisfies (3.5), we take an arbitrary $\boldsymbol{v} \in H_{0}(\operatorname{curl} ; \Omega) \cap H\left(\operatorname{div}^{0} ; \Omega ; \epsilon\right)$ and write its Hodge decomposition (cf. Lemma 2.3) as

$$
\boldsymbol{v}=\epsilon^{-1} \nabla \times \phi+\nabla \varphi
$$

where $\phi \in H^{1}(\Omega)$ and $\varphi \in \mathcal{H}(\Omega ; \epsilon)$. Note that, by Lemma 2.4, we have

$$
(\nabla \times \eta, \nabla \varphi)=0 \quad \text { and } \quad(\epsilon \boldsymbol{w}, \nabla \varphi)=(\nabla \times \rho, \nabla \varphi)=0 .
$$

It follows from (1.9), (3.6), (3.7), (3.9) and (3.10) that

$$
\begin{aligned}
\left(\mu^{-1} \nabla \times \boldsymbol{w}, \nabla \times \boldsymbol{v}\right)=(\eta, \nabla \times \boldsymbol{v}) & =(\nabla \times \eta, \boldsymbol{v}) \\
& =\left(\nabla \times \eta, \epsilon^{-1} \nabla \times \phi+\nabla \varphi\right) \\
& =\left(\nabla \times \eta, \epsilon^{-1} \nabla \times \phi\right) \\
& =-\alpha(\mu \eta, \phi) \\
& =-\alpha(\nabla \times \boldsymbol{w}, \phi) \\
& =-\alpha(\boldsymbol{w}, \nabla \times \phi) \\
& =-\alpha\left(\epsilon \boldsymbol{w}, \epsilon^{-1} \nabla \times \phi+\nabla \varphi\right)=-\alpha(\epsilon \boldsymbol{w}, \boldsymbol{v}),
\end{aligned}
$$

i.e., $\boldsymbol{w}$ satisfies (3.5).

\section{A $P_{1}$ Finite element method}

Let $\mathcal{T}_{h}$ be a quasi-uniform simplicial triangulation of $\Omega$ with mesh size $h$ and let $V_{h} \subset H^{1}(\Omega)$ be the $P_{1}$ finite element space associated with $\mathcal{T}_{h}$.

For $\alpha \neq 0$, the $P_{1}$ finite element method for (1.6) is to find $\xi_{h} \in V_{h}$ such that

$$
\left(\nabla \times \xi_{h}, \epsilon^{-1} \nabla \times v\right)+\alpha\left(\mu \xi_{h}, v\right)=\left(\boldsymbol{f}, \epsilon^{-1} \nabla \times v\right) \quad \forall v \in V_{h} .
$$

For $\alpha>0$, the problem (4.1) is symmetric positive-definite and hence well-posed. It is also well-posed for $\alpha<0$ provided $-\alpha$ is not a Maxwell eigenvalue and $h$ is sufficiently small (cf. Lemma 4.2 below).

Note that (4.1) implies

$$
\left(\mu \xi_{h}, 1\right)=0
$$

When $\Omega$ is simply connected and $\alpha=0, \xi_{h} \in V_{h}$ is determined by (4.1) together with the constraint (4.2). It is a well-posed problem because of the PoincaréFriedrichs inequality (cf. [21])

$$
\|v\|_{L_{2}(\Omega)} \leq C\left(|(\mu v, 1)|+\|\nabla \times v\|_{L_{2}(\Omega)}\right) \quad \forall v \in H^{1}(\Omega) .
$$

From here on we use $C$ (with or without subscript) to denote a generic positive constant independent of $h$, but which can depend on $\mu, \epsilon$ and $\alpha$.

The $P_{1}$ finite element approximation $\phi_{h} \in V_{h}$ of $\phi$ (cf. (1.4) $)$ is then determined by

$$
\begin{aligned}
\left(\nabla \times \phi_{h}, \epsilon^{-1} \nabla \times v\right) & =\left(\mu \xi_{h}, v\right) \quad \forall v \in V_{h}, \\
\left(\phi_{h}, 1\right) & =0 .
\end{aligned}
$$

The problem (4.4) is well-posed because of (4.2) and (4.3). 
In the case where $m \geq 1$ (i.e., $\Omega$ is not simply connected), the $P_{1}$ finite element approximation $\varphi_{j, h} \in V_{h}$ for the $\epsilon$-harmonic function $\varphi_{j}$ in the Hodge decomposition (1.2) is determined by the following problem (cf. (1.3)):

$$
\begin{array}{rlrl}
\left(\epsilon \nabla \varphi_{j, h}, \nabla v\right) & =0 & & \forall v \in \stackrel{\circ}{V}_{h}, \\
\left.\varphi_{j, h}\right|_{\Gamma_{0}} & =0, \\
\left.\varphi_{j, h}\right|_{\Gamma_{k}} & =\delta_{j k}=\left\{\begin{array}{ll}
1 & j=k, \\
0 & j \neq k,
\end{array} \text { for } \quad 1 \leq k \leq m,\right.
\end{array}
$$

where $\stackrel{\circ}{V}_{h}=V_{h} \cap H_{0}^{1}(\Omega)$ is the $P_{1}$ finite element space whose members vanish on $\partial \Omega$.

Since the bilinear form

$$
\left(\varphi_{h}, \varrho_{h}\right) \rightarrow\left(\epsilon \nabla \varphi_{h}, \nabla \varrho_{h}\right)
$$

is symmetric positive-definite on $\left\langle\varphi_{1, h}, \ldots, \varphi_{m, h}\right\rangle$, we can compute $c_{1, h}, \ldots, c_{m, h}$ by solving the symmetric positive-definite system

$$
\sum_{j=1}^{m}\left(\epsilon \nabla \varphi_{j, h}, \nabla \varphi_{k, h}\right) c_{j, h}=\frac{1}{\alpha}\left(\boldsymbol{f}, \nabla \varphi_{k, h}\right) \quad \text { for } \quad 1 \leq k \leq m .
$$

(Recall that $\alpha$ is assumed to be nonzero when $\Omega$ is not simply connected.)

Finally, we approximate $\boldsymbol{u}$ by the piecewise constant vector field $\boldsymbol{u}_{h}$ defined by

$$
\boldsymbol{u}_{h}=\epsilon^{-1} \nabla \times \phi_{h}+\sum_{j=1}^{m} c_{j, h} \nabla \varphi_{j, h}
$$

Since (4.1), (4.4) and (4.5) only involve standard second order scalar elliptic problems, the $P_{1}$ finite element method can be analyzed by standard techniques. Below is a brief error analysis where we provide details only for results that are less standard. The main theorem (Theorem 4.9) is established under the assumption that $\boldsymbol{f} \in\left[L_{2}(\Omega)\right]^{2}$. But we will also remark on various improvements on the rate of convergence under the stronger assumption that $f$ is piecewise smooth.

Let the index $\beta$ be defined by

$$
\beta=\min \left(1, \min _{1 \leq \ell \leq L} \frac{\pi}{\omega_{\ell}}\right),
$$

where $\omega_{1}, \omega_{2}, \ldots, \omega_{L}$ are the interior angles at the corners of $\Omega$. Note that $\beta=1$ if $\Omega$ is convex.

The following estimate for the solution of (1.3) can be derived from the singular function representations of these solutions (cf. [15, 13, 20]) and standard interpolation error estimates [11, 9]:

$$
\left\|\varphi_{j}-\Pi_{h} \varphi_{j}\right\|_{L_{2}(\Omega)}+h\left|\varphi_{j}-\Pi_{h} \varphi_{j}\right|_{H^{1}(\Omega)} \leq C h^{1+\beta},
$$

where $\Pi_{h}$ is the nodal interpolation operator for the $P_{1}$ finite element.

Similarly, for the solution $\zeta$ of the Laplace equation with homogeneous Neumann boundary condition, we have

$$
\left\|\zeta-\Pi_{h} \zeta\right\|_{L_{2}(\Omega)}+h\left|\zeta-\Pi_{h} \zeta\right|_{H^{1}(\Omega)} \leq C h^{1+\beta}\|g\|_{L_{2}(\Omega)},
$$

where $g$ is the right-hand side function.

We begin by comparing $\xi_{h}$ and $\xi=\mu^{-1} \nabla \times \boldsymbol{u}$. The following result is obtained by using (1.6), (4.1), (4.10) and a standard duality argument. 
Lemma 4.1. For $\alpha>0($ general $\Omega)$ and $\alpha=0$ (simply connected $\Omega)$, we have

$$
\left\|\xi-\xi_{h}\right\|_{L_{2}(\Omega)} \leq C h^{\beta} \inf _{v \in V_{h}}\|\nabla \times(\xi-v)\|_{L_{2}(\Omega)} .
$$

In the case where $\alpha<0$, the following result is obtained by using the approach of Schatz 23], where the required well-posedness of the continuous problem (1.6) is guaranteed by Lemma 3.2

Lemma 4.2. The discrete problem (4.1) is well-posed for $\alpha<0$, provided $-\alpha$ is not a Maxwell eigenvalue and $h$ is sufficiently small. Under these conditions the estimate (4.11) remains valid.

Under the assumption that $\boldsymbol{f} \in\left[L_{2}(\Omega)\right]^{2}$, we have the following stability estimate from the well-posedness of the continuous problem:

$$
\|\xi\|_{H^{1}(\Omega)} \leq C\|\boldsymbol{f}\|_{L_{2}(\Omega)},
$$

which together with (4.11) immediately implies the following corollary.

Corollary 4.3. Under the conditions in Lemmas 4.1 and 4.2, we have

$$
\left\|\xi-\xi_{h}\right\|_{L_{2}(\Omega)} \leq C h^{\beta}\|\boldsymbol{f}\|_{L_{2}(\Omega)} .
$$

Remark 4.4. If $\boldsymbol{f}$ is a piecewise smooth vector field, then it follows from integration by parts and the trace theorem that

$$
\left|\left(\boldsymbol{f}, \epsilon^{-1} \nabla \times v\right)\right| \leq C_{\delta}\|v\|_{H^{(1 / 2)+\delta}(\Omega)} \quad \forall v \in H^{1}(\Omega),
$$

where $\delta>0$ is arbitrary. Hence we have $\xi \in H^{(3 / 2)-\delta}(\Omega)$ by elliptic regularity and it follows from (4.11) that the estimate (4.13) can be improved to

$$
\left\|\xi-\xi_{h}\right\|_{L_{2}(\Omega)} \leq C_{\delta} h^{\beta+(1 / 2)-\delta} .
$$

Next, we compare $\phi_{h}$ and $\phi$.

Lemma 4.5. For $h$ sufficiently small, we have

$\left\|\nabla \times\left(\phi-\phi_{h}\right)\right\|_{L_{2}(\Omega)} \leq C\left(h^{\beta} \inf _{v \in V_{h}}\|\nabla \times(\xi-v)\|_{L_{2}(\Omega)}+\inf _{v \in V_{h}}\|\nabla \times(\phi-v)\|_{L_{2}(\Omega)}\right)$.

Proof. Since $(\mu \xi, 1)=0$, we can define $\tilde{\phi}_{h} \in V_{h}$ to be the unique solution of

$$
\begin{aligned}
\left(\nabla \times \tilde{\phi}_{h}, \epsilon^{-1} \nabla \times v\right) & =(\mu \xi, v) \quad \forall v \in V_{h}, \\
\left(\tilde{\phi}_{h}, 1\right) & =0 .
\end{aligned}
$$

It follows from (4.4) and (4.16) that

$$
\left(\nabla \times\left(\tilde{\phi}_{h}-\phi_{h}\right), \epsilon^{-1} \nabla \times v\right)=\left(\mu\left(\xi-\xi_{h}\right), v\right) \quad \forall v \in V_{h},
$$

and $\left(\phi_{h}-\tilde{\phi}_{h}, 1\right)=0$. We then obtain, by (4.3), (4.11) and (4.17),

$$
\begin{aligned}
\left\|\epsilon^{-\frac{1}{2}} \nabla \times\left(\tilde{\phi}_{h}-\phi_{h}\right)\right\|_{L_{2}(\Omega)}^{2} & =\left(\mu\left(\xi-\xi_{h}\right), \tilde{\phi}_{h}-\phi_{h}\right) \\
& \leq C\left\|\xi-\xi_{h}\right\|_{L_{2}(\Omega)}\left\|\tilde{\phi}_{h}-\phi_{h}\right\|_{L_{2}(\Omega)} \\
& \leq C h^{\beta} \inf _{v \in V_{h}}\|\nabla \times(\xi-v)\|_{L_{2}(\Omega)}\left\|\nabla \times\left(\tilde{\phi}_{h}-\phi_{h}\right)\right\|_{L_{2}(\Omega)},
\end{aligned}
$$

which implies

$$
\left\|\nabla \times\left(\tilde{\phi}_{h}-\phi_{h}\right)\right\|_{L_{2}(\Omega)} \leq C h^{\beta} \inf _{v \in V_{h}}\|\nabla \times(\xi-v)\|_{L_{2}(\Omega)} .
$$


Comparing (1.4) and (4.16a), we have the Galerkin relation

$$
\left(\nabla \times\left(\phi-\tilde{\phi}_{h}\right), \epsilon^{-1} \nabla \times v\right)=0 \quad \forall v \in V_{h},
$$

which implies

$$
\left\|\epsilon^{-\frac{1}{2}} \nabla \times\left(\phi-\tilde{\phi}_{h}\right)\right\|_{L_{2}(\Omega)}=\inf _{v \in V_{h}}\left\|\epsilon^{-\frac{1}{2}} \nabla \times(\phi-v)\right\|_{L_{2}(\Omega)} .
$$

The estimate (4.15) follows from (4.18) and (4.19).

Note that (1.4), (1.5) and (4.10) imply

$$
\inf _{v \in V_{h}}\|\nabla \times(\phi-v)\|_{L_{2}(\Omega)} \leq\left\|\nabla \times\left(\phi-\Pi_{h} \phi\right)\right\|_{L_{2}(\Omega)} \leq C h^{\beta}\|\xi\|_{L_{2}(\Omega)} .
$$

Hence, under the assumption that $f \in\left[L_{2}(\Omega)\right]^{2}$, we can use (4.12), (4.15) and (4.20) to obtain the following bound:

$$
\left\|\nabla \times\left(\phi-\phi_{h}\right)\right\|_{L_{2}(\Omega)} \leq C h^{\beta}\|\boldsymbol{f}\|_{L_{2}(\Omega)} .
$$

The next result follows from a standard argument using (4.9) and Galerkin orthogonality.

Lemma 4.6. We have, for $1 \leq j \leq m$,

$$
\left|\varphi_{j}-\varphi_{j, h}\right|_{H^{1}(\Omega)} \leq C h^{\beta} .
$$

Next we compare $c_{j, h}$ and $c_{j}$. First we observe that (4.22) implies

$$
\left|\left(\boldsymbol{f}, \nabla \varphi_{j}\right)-\left(\boldsymbol{f}, \nabla \varphi_{j, h}\right)\right| \leq C h^{\beta}\|\boldsymbol{f}\|_{L_{2}(\Omega)} \quad \text { for } 1 \leq j \leq m .
$$

Furthermore, since $\varphi_{i}-\varphi_{i, h} \in H_{0}^{1}(\Omega)$ for $1 \leq i \leq m$, (1.3a) implies

$\left(\epsilon \nabla \varphi_{i}, \nabla \varphi_{j}\right)-\left(\epsilon \nabla \varphi_{i, h}, \nabla \varphi_{j, h}\right)=\left(\epsilon\left(\nabla \varphi_{i}-\nabla \varphi_{i, h}\right), \nabla \varphi_{j, h}-\nabla \varphi_{j}\right) \quad$ for $1 \leq i, j \leq m$, and hence, in view of (4.22),

$$
\left|\left(\epsilon \nabla \varphi_{i}, \nabla \varphi_{j}\right)-\left(\epsilon \nabla \varphi_{i, h}, \nabla \varphi_{j, h}\right)\right| \leq C h^{2 \beta} \quad \text { for } 1 \leq i, j \leq m .
$$

Lemma 4.7. For $h$ sufficiently small, we have

$$
\left|c_{j}-c_{j, h}\right| \leq C h^{\beta}\|\boldsymbol{f}\|_{L_{2}(\Omega)} \quad \text { for } \quad 1 \leq j \leq m .
$$

Proof. We can write (1.8) and (4.6) as

$$
\mathbf{A c}=\mathbf{b} \quad \text { and } \quad \mathbf{A}_{h} \mathbf{c}_{h}=\mathbf{b}_{h},
$$

where $\mathbf{c} \in \mathbb{R}^{\mathbf{m}}$ (resp. $\mathbf{c}_{h} \in \mathbb{R}^{m}$ ) is the vector whose $j$-th component is $c_{j}$ (resp. $c_{j, h}$ ), $\mathbf{A} \in \mathbb{R}^{m \times m}$ (resp. $\mathbf{A}_{h} \in \mathbb{R}^{m \times m}$ ) is the matrix whose $(i, j)$-th component is $\left(\epsilon \nabla \varphi_{j}, \nabla \varphi_{i}\right)$ (resp. $\left.\left(\epsilon \nabla \varphi_{j, h}, \nabla \varphi_{i, h}\right)\right)$, and $\mathbf{b} \in \mathbb{R}^{m}$ (resp. $\left.\mathbf{b}_{h} \in \mathbb{R}^{m}\right)$ is the vector whose $j$-th component is $\alpha^{-1}\left(\boldsymbol{f}, \nabla \varphi_{j}\right)$ (resp. $\left.\alpha^{-1}\left(\boldsymbol{f}, \nabla \varphi_{j, h}\right)\right)$.

Note that

$$
\|\mathbf{b}\|_{\infty} \leq|\alpha|^{-1}\left(\max _{1 \leq j \leq m}\left\|\nabla \varphi_{j}\right\|_{L_{2}(\Omega)}\right)\|\boldsymbol{f}\|_{L_{2}(\Omega)} \leq C\|\boldsymbol{f}\|_{L_{2}(\Omega)},
$$

and the estimates (4.23)-(4.24) are translated into

$$
\left\|\mathbf{b}-\mathbf{b}_{h}\right\|_{\infty} \leq C h^{\beta}\|\boldsymbol{f}\|_{L_{2}(\Omega)} \quad \text { and } \quad\left\|\mathbf{A}-\mathbf{A}_{h}\right\|_{\infty} \leq C h^{2 \beta} .
$$

The estimate (4.25) follows from the identity

$$
\mathbf{c}-\mathbf{c}_{h}=\mathbf{A}^{-1} \mathbf{b}-\mathbf{A}_{h}^{-1} \mathbf{b}_{h}=\mathbf{A}^{-1}\left(\mathbf{b}-\mathbf{b}_{h}\right)+\mathbf{A}^{-1}\left(\mathbf{A}_{h}-\mathbf{A}\right) \mathbf{A}_{h}^{-1}\left(\left(\mathbf{b}_{h}-\mathbf{b}\right)+\mathbf{b}\right)
$$
and (4.26)-(4.27). 
Remark 4.8. In the case where $\boldsymbol{f}$ is piecewise smooth, it follows from integration by parts and the trace theorem that

$$
\left|\left(\boldsymbol{f}, \nabla \varphi_{j}\right)-\left(\boldsymbol{f}, \nabla \varphi_{j, h}\right)\right| \leq C_{\delta}\left\|\varphi_{j}-\varphi_{j, h}\right\|_{H^{(1 / 2)+\delta}(\Omega)}
$$

for any $\delta>0$, and by a duality argument, we have

$$
\left\|\varphi_{j}-\varphi_{j, h}\right\|_{H^{(1 / 2)+\delta}(\Omega)} \leq C_{\delta} h^{(1 / 2)-\delta}\left|\varphi_{j}-\varphi_{j, h}\right|_{H^{1}(\Omega)} .
$$

Hence the estimate (4.23) can be improved to

$$
\left|\left(\boldsymbol{f}, \nabla \varphi_{j}\right)-\left(\boldsymbol{f}, \nabla \varphi_{j, h}\right)\right| \leq C_{\delta} h^{\beta+(1 / 2)-\delta},
$$

and we can replace (4.25) by

$$
\left|c_{j}-c_{j, h}\right| \leq C_{\delta} h^{\beta+(1 / 2)-\delta} \quad \text { for } \quad 1 \leq j \leq m .
$$

We can now compare $\boldsymbol{u}_{h}$ and $\boldsymbol{u}$ by putting all the estimates together.

Theorem 4.9. For $h$ sufficiently small, we have

$$
\left\|\boldsymbol{u}-\boldsymbol{u}_{h}\right\|_{L_{2}(\Omega)} \leq C h^{\beta}\|\boldsymbol{f}\|_{L_{2}(\Omega)} .
$$

Proof. First we observe that the solutions $c_{1}, \ldots, c_{m}$ of (1.8) satisfy

$$
\left|c_{j}\right| \leq C\|\boldsymbol{f}\|_{L_{2}(\Omega)} \quad \text { for } \quad 1 \leq j \leq m .
$$

Second we have, from (1.2) and (4.7),

$$
\begin{aligned}
\| \boldsymbol{u}- & \boldsymbol{u}_{h} \|_{L_{2}(\Omega)} \leq C\left|\phi-\phi_{h}\right|_{H^{1}(\Omega)}+\sum_{j=1}^{m}\left|c_{j} \varphi_{j}-c_{j, h} \varphi_{j, h}\right|_{H^{1}(\Omega)} \\
\leq & C\left|\phi-\phi_{h}\right|_{H^{1}(\Omega)}+\sum_{j=1}^{m}\left(\left|c_{j}-c_{j, h}\right|\left|\varphi_{j}\right|_{H^{1}(\Omega)}+\left|c_{j, h}\right|\left|\varphi_{j}-\varphi_{j, h}\right|_{H^{1}(\Omega)}\right) \\
\leq & C\left|\phi-\phi_{h}\right|_{H^{1}(\Omega)}+\sum_{j=1}^{m}\left|c_{j}-c_{j, h}\right|\left(\left|\varphi_{j}\right|_{H^{1}(\Omega)}+\left|\varphi_{j}-\varphi_{j, h}\right|_{H^{1}(\Omega)}\right) \\
& +\sum_{j=1}^{m}\left|c_{j}\right|\left|\varphi_{j}-\varphi_{j, h}\right|_{H^{1}(\Omega)} .
\end{aligned}
$$

The estimate (4.29) follows from (4.21), (4.22), (4.25), (4.30) and (4.31).

Remark 4.10. In the case where $c_{j}=0=c_{j, h}$ for $1 \leq j \leq m$, it follows from (4.15) and (4.31) that

$$
\left\|\boldsymbol{u}-\boldsymbol{u}_{h}\right\|_{L_{2}(\Omega)} \leq C\left[h^{\beta} \inf _{v \in V_{h}}\|\nabla \times(\xi-v)\|_{L_{2}(\Omega)}+\inf _{v \in V_{h}}\|\nabla \times(\phi-v)\|_{L_{2}(\Omega)}\right] .
$$

Finally, we present the results of several numerical tests that illustrate the performance of the $P_{1}$ finite element method. We take $\mu=\epsilon=1$ in the tests. All the computations are performed on uniform grids consisting of isosceles right-angled triangles whose horizontal and vertical edges have length $h$. 
TABLE 4.1. Results for (1.1) on the L-shaped domain with exact solution given by (4.32)

\begin{tabular}{|c|c|c|c|c|c|}
\hline$h$ & $\frac{\left\|\nabla \times \boldsymbol{u}-\xi_{h}\right\|_{L_{2}}}{\|\mathbf{f}\|_{L_{2}}}$ & Order & $h$ & $\frac{\left\|\boldsymbol{u}-\boldsymbol{u}_{h}\right\|_{L_{2}}}{\|\mathbf{f}\|_{L_{2}}}$ & Order \\
\hline \multicolumn{6}{|c|}{$\alpha=-1$} \\
\hline $1 / 8$ & $3.57 \mathrm{E}-02$ & 1.43 & $1 / 8$ & $3.19 \mathrm{E}-02$ & 1.41 \\
$1 / 16$ & $1.32 \mathrm{E}-02$ & 1.43 & $1 / 16$ & $1.23 \mathrm{E}-02$ & 1.38 \\
$1 / 32$ & $4.98 \mathrm{E}-03$ & 1.41 & $1 / 32$ & $5.03 \mathrm{E}-03$ & 1.28 \\
$1 / 64$ & $1.90 \mathrm{E}-03$ & 1.39 & $1 / 64$ & $2.26 \mathrm{E}-03$ & 1.15 \\
$1 / 128$ & $7.37 \mathrm{E}-04$ & 1.37 & $1 / 128$ & $1.13 \mathrm{E}-03$ & 0.99 \\
$1 / 256$ & $2.87 \mathrm{E}-04$ & 1.36 & $1 / 256$ & $6.17 \mathrm{E}-04$ & 0.87 \\
\hline \multicolumn{6}{|c|}{$\alpha=0$} \\
\hline $1 / 8$ & $1.12 \mathrm{E}-02$ & 1.44 & $1 / 8$ & $1.35 \mathrm{E}-02$ & 1.29 \\
$1 / 16$ & $4.24 \mathrm{E}-03$ & 1.41 & $1 / 64$ & $6.13 \mathrm{E}-03$ & 1.14 \\
$1 / 32$ & $1.63 \mathrm{E}-03$ & 1.38 & $1 / 32$ & $3.07 \mathrm{E}-03$ & 0.99 \\
$1 / 64$ & $6.36 \mathrm{E}-04$ & 1.36 & $1 / 64$ & $1.66 \mathrm{E}-03$ & 0.89 \\
$1 / 128$ & $2.50 \mathrm{E}-04$ & 1.35 & $1 / 128$ & $9.46 \mathrm{E}-04$ & 0.81 \\
$1 / 256$ & $9.86 \mathrm{E}-05$ & 1.34 & $1 / 256$ & $5.58 \mathrm{E}-04$ & 0.76 \\
\hline \multicolumn{7}{|c|}{$\alpha=1$} \\
\hline $1 / 8$ & $6.77 \mathrm{E}-03$ & 1.39 & $1 / 8$ & $1.06 \mathrm{E}-02$ & 1.14 \\
$1 / 16$ & $2.63 \mathrm{E}-03$ & 1.36 & $1 / 16$ & $5.27 \mathrm{E}-03$ & 1.01 \\
$1 / 32$ & $1.04 \mathrm{E}-03$ & 1.34 & $1 / 32$ & $2.80 \mathrm{E}-03$ & 0.91 \\
$1 / 64$ & $4.14 \mathrm{E}-04$ & 1.33 & $1 / 64$ & $1.56 \mathrm{E}-03$ & 0.84 \\
$1 / 128$ & $1.65 \mathrm{E}-04$ & 1.33 & $1 / 128$ & $9.06 \mathrm{E}-04$ & 0.79 \\
$1 / 256$ & $6.57 \mathrm{E}-05$ & 1.32 & $1 / 256$ & $5.38 \mathrm{E}-04$ & 0.75 \\
\hline
\end{tabular}

In the first set of experiments, we examine the convergence behavior of the numerical scheme on the L-shaped domain $(-1,1)^{2} \backslash[0,1]^{2}$. The exact solution is chosen to be

$$
\boldsymbol{u}=\nabla \times\left(r^{2 / 3} \cos \left(\frac{2}{3} \theta-\frac{\pi}{3}\right) \phi(x)\right),
$$

where $(r, \theta)$ are the polar coordinates at the origin and $\phi(x)=\left(1-x_{1}^{2}\right)^{2}\left(1-x_{2}^{2}\right)^{2}$. It has the correct Maxwell singularity at the reentrant corner. We solve (1.1) for $\alpha=-1,0$ and 1 , with $\boldsymbol{f}=\nabla \times(\nabla \times \boldsymbol{u})-\alpha \boldsymbol{u} \in H\left(\operatorname{div}^{0} ; \Omega\right)$. The results are tabulated in Table 4.1.

Note that the convergence of $\boldsymbol{u}_{h}$ to $\boldsymbol{u}$ is approaching the order of $\beta=2 / 3$ predicted by Theorem 4.9. On the other hand, since $\xi=\nabla \times \boldsymbol{u}$ behaves like $r^{2 / 3}$ at the origin, the order of convergence for $\xi_{h}$ according to (4.11) is $(2 / 3)+(2 / 3)=4 / 3$, which agrees with the observed order of convergence.

The second set of experiments is performed for the doubly connected domain

$$
\Omega=(0,4)^{2} \backslash[1,3]^{2} .
$$

In this case the solution $\boldsymbol{u}$ of (1.1) can be written as

$$
\boldsymbol{u}=\nabla \times \phi+c \nabla \varphi
$$


TABLE 4.2. Results for (1.1) on the doubly connected domain with exact solution given by (4.34)

\begin{tabular}{|c|c|c|c|c|c|}
\hline$h$ & $\frac{\left\|\nabla \times \boldsymbol{u}-\xi_{h}\right\|_{L_{2}}}{\|\mathbf{f}\|_{L_{2}}}$ & Order & $\left|c_{h}\right|$ & $\frac{\left\|\boldsymbol{u}-\boldsymbol{u}_{h}\right\|_{L_{2}}}{\|\mathbf{f}\|_{L_{2}}}$ & Order \\
\hline \multicolumn{6}{|c|}{$\alpha=-1$} \\
\hline $1 / 8$ & $3.71 \mathrm{E}-03$ & 2.01 & $7.93 \mathrm{E}-17$ & $1.13 \mathrm{E}-03$ & 1.05 \\
$1 / 16$ & $9.26 \mathrm{E}-04$ & 2.00 & $1.36 \mathrm{E}-16$ & $5.61 \mathrm{E}-03$ & 1.01 \\
$1 / 32$ & $2.31 \mathrm{E}-04$ & 2.00 & $1.49 \mathrm{E}-16$ & $2.80 \mathrm{E}-03$ & 1.00 \\
$1 / 64$ & $5.78 \mathrm{E}-05$ & 2.00 & $7.69 \mathrm{E}-16$ & $1.39 \mathrm{E}-03$ & 1.00 \\
$1 / 128$ & $1.44 \mathrm{E}-05$ & 2.00 & $7.43 \mathrm{E}-16$ & $6.99 \mathrm{E}-04$ & 1.00 \\
\hline \multicolumn{7}{|c|}{$\alpha=1$} \\
\hline $1 / 8$ & $1.69 \mathrm{E}-03$ & 1.98 & $9.25 \mathrm{E}-16$ & $9.50 \mathrm{E}-03$ & 1.00 \\
$1 / 16$ & $4.25 \mathrm{E}-04$ & 1.99 & $1.11 \mathrm{E}-15$ & $4.75 \mathrm{E}-03$ & 1.00 \\
$1 / 32$ & $1.06 \mathrm{E}-04$ & 2.00 & $1.35 \mathrm{E}-15$ & $2.38 \mathrm{E}-03$ & 1.00 \\
$1 / 64$ & $2.66 \mathrm{E}-05$ & 2.00 & $3.27 \mathrm{E}-15$ & $1.19 \mathrm{E}-03$ & 1.00 \\
$1 / 128$ & $6.64 \mathrm{E}-06$ & 2.00 & $4.96 \mathrm{E}-15$ & $5.94 \mathrm{E}-04$ & 1.00 \\
\hline
\end{tabular}

where $c$ is a constant and the harmonic function $\varphi$ satisfies the boundary conditions

$$
\left.\varphi\right|_{\Gamma_{0}}=0 \text { and }\left.\varphi\right|_{\Gamma_{1}}=1 .
$$

Here $\Gamma_{0}$ (resp. $\Gamma_{1}$ ) is the boundary of $(0,4)^{2}$ (resp. $\left.(1,3)^{2}\right)$. First, we take the exact solution to be

$$
\boldsymbol{u}=\left[\begin{array}{l}
x_{2}\left(1-x_{2}\right)\left(3-x_{2}\right)\left(4-x_{2}\right) \\
x_{1}\left(1-x_{1}\right)\left(3-x_{1}\right)\left(4-x_{1}\right)
\end{array}\right]
$$

and solve (1.1) for $\alpha=-1$ and 1 , with $\boldsymbol{f}=\nabla \times(\nabla \times \boldsymbol{u})-\alpha \boldsymbol{u} \in H\left(\operatorname{div}^{0} ; \Omega\right)$. The numerical results are presented in Table 4.2 .

Note that in this case $\boldsymbol{u}$ is the curl of a quintic polynomial and hence $c=0$ in (4.33). In fact, since $\boldsymbol{f}$ is also the curl of a polynomial, we have $\left(\boldsymbol{f}, \nabla \varphi_{h}\right)=0$ by Lemma 2.4 and it is observed that $c_{h}=0$ up to machine error.

According to Remark 4.10, the order of convergence for $\boldsymbol{u}_{h}$ is 1 (since $\xi$ and $\phi$ are smooth), which is observed. The order of convergence for $\xi_{h}$ is found to be 2 , which is better than the order of $\beta+1=5 / 3$ predicted by (4.11). This is likely due to the effects of superconvergence [24, 18] since we use uniform meshes in computing $\xi_{h}$ and the exact solution $\xi$ is smooth.

Finally, we take the right-hand side of (1.1) to be the piecewise smooth vector field

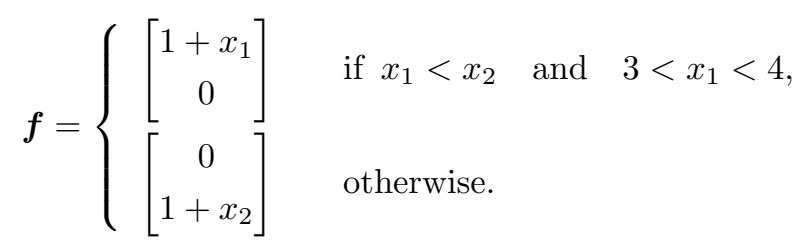

The numerical results are presented in Table 4.3 for $\alpha=-1$ and 1 . 
TABLE 4.3. Results for (1.1) on the doubly connected domain with right-hand side given by (4.35)

\begin{tabular}{|c|c|c|c|c|c|c|}
\hline$h$ & $\frac{\left\|\nabla \times \boldsymbol{u}-\xi_{h}\right\|_{L_{2}}}{\|\mathbf{f}\|_{L_{2}}}$ & Order & $c_{h}$ & Order & $\frac{\left\|\boldsymbol{u}-\boldsymbol{u}_{h}\right\|_{L_{2}}}{\|\mathbf{f}\|_{L_{2}}}$ & Order \\
\hline \multicolumn{7}{|c|}{$\alpha=-1$} \\
\hline $1 / 4$ & $1.72 \mathrm{E}-01$ & 1.26 & 0.763918 & 1.05 & $2.68 \mathrm{E}-01$ & 1.00 \\
$1 / 8$ & $5.28 \mathrm{E}-02$ & 1.70 & 0.765285 & 0.87 & $1.28 \mathrm{E}-01$ & 1.06 \\
$1 / 16$ & $1.49 \mathrm{E}-02$ & 1.83 & 0.765991 & 0.95 & $6.93 \mathrm{E}-02$ & 0.89 \\
$1 / 32$ & $4.29 \mathrm{E}-03$ & 1.80 & 0.766332 & 1.05 & $4.04 \mathrm{E}-02$ & 0.78 \\
$1 / 64$ & $1.13 \mathrm{E}-03$ & 1.69 & 0.766489 & 1.12 & $2.42 \mathrm{E}-02$ & 0.73 \\
\hline \multicolumn{7}{|c|}{$\alpha=1$} \\
\hline $1 / 4$ & $1.03 \mathrm{E}-02$ & 1.33 & -0.763918 & 1.05 & $8.60 \mathrm{E}-02$ & 0.71 \\
$1 / 8$ & $4.04 \mathrm{E}-03$ & 1.35 & -0.765285 & 0.87 & $5.30 \mathrm{E}-02$ & 0.70 \\
$1 / 16$ & $1.58 \mathrm{E}-03$ & 1.35 & -0.765991 & 0.95 & $3.29 \mathrm{E}-02$ & 0.69 \\
$1 / 32$ & $6.21 \mathrm{E}-04$ & 1.35 & -0.766332 & 1.05 & $2.05 \mathrm{E}-02$ & 0.68 \\
$1 / 64$ & $2.44 \mathrm{E}-04$ & 1.34 & -0.766489 & 1.12 & $1.28 \mathrm{E}-02$ & 0.67 \\
\hline
\end{tabular}

The observed orders of convergence are consistent with the theoretical results. In particular, the order of convergence for $c_{h}$ matches the estimate (4.28) with $\beta=2 / 3$, and the order of convergence for $\boldsymbol{u}_{h}$ is $2 / 3$ for $\alpha=1$ and approaching $2 / 3$ for $\alpha=-1$, which agrees with the estimate (4.29). The order of convergence for $\xi_{h}$ in both cases is higher than the order predicted by (4.14). This is probably due to the fact that the mesh size $h$ is not small enough and the asymptotic behavior has not been reached.

\section{Concluding REMARKS}

The new numerical approach for two-dimensional Maxwell's equations introduced in this paper only involves solving standard second order scalar elliptic boundary value problems. We have demonstrated its performance using a $P_{1}$ finite element method on quasi-uniform triangulations. There are of course many other possibilities, such as finite element methods on graded meshes that can recover $O(h)$ convergence even on nonconvex domains, adaptive methods, multigrid methods and domain decomposition methods. The application of these methods to (1.1) and the related Maxwell eigenproblem will be carried out elsewhere.

We can also apply the Hodge decomposition to study Maxwell's equations in three dimensions. In this case both $\boldsymbol{\xi}=\nabla \times \boldsymbol{u}$ and the potential $\boldsymbol{\phi}$ in the Hodge decomposition are vector fields. Therefore, the Hodge decomposition does not reduce the problem to scalar elliptic boundary value problems. However, there may still be some advantages of solving the systems for $\boldsymbol{\xi}$ and $\phi$ instead of the original system for $\boldsymbol{u}$. This will be further explored.

\section{ACKNOWLEDGEMENT}

Part of the research in this paper was carried out when the first author was visiting Université Pierre et Marie Curie. She would like to thank the members of the Laboratoire Jacques-Louis Lions for their hospitality. 


\section{REFERENCES}

[1] R.A. Adams and J.J.F. Fournier. Sobolev Spaces (Second Edition). Academic Press, Amsterdam, 2003. MR2424078 (2009e:46025)

[2] A. Alonso, P. Fernandes, and A. Valli. Weak and strong formulations for the time-harmonic eddy-current problem in general multi-connected domains. European J. Appl. Math., 14:387406, 2003. MR.1999131 (2004i:78003)

[3] A. Alonso-Rodríguez, A. Valli, and R. Vázquez-Hernández. A formulation of the eddy current problem in the presence of electric ports. Numer. Math., 113:643-672, 2009. MR2545497 (2010k:65251)

[4] C. Amrouche, C. Bernardi, M. Dauge, and V. Girault. Vector potentials in three-dimensional non-smooth domains. Math. Methods Appl. Sci., 21:823-864, 1998. MR.1626990 (99e:35037)

[5] F. Assous and M. Michaeli. Hodge decomposition to solve singular static Maxwell's equations in a non-convex polygon. Appl. Numer. Math., 60:432-441, 2010. MR2607801(2011c:65251)

[6] S.C. Brenner, F. Li, and L.-Y. Sung. A locally divergence-free nonconforming finite element method for the time-harmonic Maxwell equations. Math. Comp., 76:573-595, 2007. MR2291828(2008c:65316)

[7] S.C. Brenner, F. Li, and L.-Y. Sung. A locally divergence-free interior penalty method for two dimensional curl-curl problems. SIAM J. Numer. Anal., 46:1190-1211, 2008. MR2390990 (2009d:35321)

[8] S.C. Brenner, F. Li, and L.-Y. Sung. A nonconforming penalty method for a two dimensional curl-curl problem. M3AS, 19:651-668, 2009. MR2514440(2010h:65222)

[9] S.C. Brenner and L.R. Scott. The Mathematical Theory of Finite Element Methods (Third Edition). Springer-Verlag, New York, 2008. MR 2373954 (2008m:65001)

[10] P. Ciarlet, Jr. Augmented formulations for solving Maxwell equations. Comput. Methods Appl. Mech. Engrg., 194:559-586, 2005. MR2105182 (2005h:78026)

[11] P.G. Ciarlet. The Finite Element Method for Elliptic Problems. North-Holland, Amsterdam, 1978. MR0520174 (58:25001)

[12] M. Costabel and M. Dauge. Maxwell and Lamé eigenvalues on polyhedra. Math. Methods Appl. Sci., 22:243-258, 1999. MR.1672271 (99k:78009)

[13] M. Dauge. Elliptic Boundary Value Problems on Corner Domains, Lecture Notes in Mathematics 1341. Springer-Verlag, Berlin-Heidelberg, 1988. MR.961439 (91a:35078)

[14] V. Girault and P.-A. Raviart. Finite Element Methods for Navier-Stokes Equations. Theory and Algorithms. Springer-Verlag, Berlin, 1986. MR851383 (88b:65129)

[15] P. Grisvard. Elliptic Problems in Nonsmooth Domains. Pitman, Boston, 1985. MR 0775683 (86n:35044)

[16] R. Hiptmair. Finite elements in computational electromagnetism. Acta Numer., 11:237-339, 2002. MR2009375 (2004k:78028)

[17] R. Hiptmair and J. Xu. Nodal auxiliary space preconditioning in $\mathbf{H}(\mathbf{c u r l})$ and $\mathbf{H}$ (div) spaces. SIAM J. Numer. Anal., 45:2483-2509 (electronic), 2007. MR2361899 (2009g:65153)

[18] Q. Lin and J. Lin. Finite Element Methods: Accuracy and Improvement. Science Press, Beijing, 2006.

[19] P. Monk. Finite Element Methods for Maxwell's Equations. Oxford University Press, New York, 2003. MR2059447 (2005d:65003)

[20] S.A. Nazarov and B.A. Plamenevsky. Elliptic Problems in Domains with Piecewise Smooth Boundaries. de Gruyter, Berlin-New York, 1994. MR1283387 (95h:35001)

[21] J. Nečas. Les Méthodes Directes en Théorie des Équations Elliptiques. Masson, Paris, 1967. MR0227584 (37:3168)

[22] J. E. Pasciak and J. Zhao. Overlapping Schwarz methods in $H$ (curl) on polyhedral domains. J. Numer. Math., 10:221-234, 2002. MR 1935967(2003j:65108)

[23] A. Schatz. An observation concerning Ritz-Galerkin methods with indefinite bilinear forms. Math. Comp., 28:959-962, 1974. MR0373326 (51:9526)

[24] L.B. Wahlbin. Superconvergence in Galerkin Finite Element Methods, Lecture Notes in Mathematics 1605. Springer-Verlag, Berlin, 1995. MR1439050 (98j:65083)

[25] C. Weber. A local compactness theorem for Maxwell's equations. Math. Meth. Appl. Sci., 2:12-25, 1980. MR561375 (81f:78005) 
Department of Mathematics and Center for Computation and Technology, Louisiana State University, Baton Rouge, Louisiana 70803

E-mail address: brenner@math.1su.edu

Department of Mathematics and Center for Computation and Technology, louisiana State University, Baton Rouge, Louisiana 70803

Current address: Institute for Mathematics and its Applications, University of Minnesota, Minneapolis, Minnesota 55455

E-mail address: jcui@ima.umn.edu

Department of Mathematics and Center for Computation and Technology, louisiana State University, Baton Rouge, Louisiana 70803

E-mail address: zhenan@math.lsu.edu

Department of Mathematics and Center for Computation and Technology, louisiana State University, Baton Rouge, Louisiana 70803

E-mail address: sung@math.1su.edu 